\title{
ON PASZKIEWICZ-TYPE CRITERION FOR A.E. CONTINUITY OF PROCESSES IN $L^{p}$-SPACES
}

\author{
JAKUB OLEJNIK \\ Faculty of Mathematics and Computer Science, University of Eódź \\ Banacha 22, 90-238 Eódź, Poland \\ E-mail: jakubo@math.uni.lodz.pl
}

\begin{abstract}
In this paper we consider processes $X_{t}$ with values in $L^{p}, p \geq 1$ on subsets $T$ of a unit cube in $\mathbb{R}^{n}$ satisfying a natural condition of boundedness of increments, i.e. a process has bounded increments if for some non-decreasing $f: \mathbb{R}_{+} \rightarrow \mathbb{R}_{+}$

$$
\left\|X_{t}-X_{s}\right\|_{p} \leq f(\|t-s\|), \quad s, t \in T .
$$

We give a sufficient criterion for a.s. continuity of all processes with bounded increments on subsets of a given set $T$. This criterion turns out to be necessary for a wide class of functions $f$. We use a geometrical Paszkiewicz-type characteristic of the set $T$. Our result generalizes in some way the classical theorem by Kolmogorov.
\end{abstract}

1. Introduction. In this paper we investigate conditions of almost sure continuity of processes with 'bounded increments' in $L_{p}$ spaces, for $p \geq 1$. For a fixed probability space and a non-decreasing function $f: \mathbb{R}_{+} \rightarrow \mathbb{R}_{+}$we will say that a process $\left(X_{t}\right)_{t \in T}$ on a subset $T$ of the unit cube in $\mathbb{R}^{\eta}$ (with $\eta$ fixed) has bounded increments if

$$
\forall_{s, t \in T} \quad\left\|X_{t}-X_{s}\right\|_{p} \leq f\left(d_{\infty}(t, s)\right) .
$$

More precisely, sets $T \subset[0,1]^{\eta}, \eta \geq 1$ are considered, and $d_{\infty}(s, t)=\max _{1 \leq i \leq \eta}\left|s_{i}-t_{i}\right|$, for $s=\left(s_{1}, \ldots, s_{\eta}\right), t=\left(t_{1}, \ldots, t_{\eta}\right)$ in $\mathbb{R}^{\eta}$. It is merely a matter of convenience to use $d_{\infty}$ instead of the natural Euclidean metric. We give a condition on $T$ which is sufficient for existence of a.e.-continuous version of every process $\left(X_{t}\right)_{t \in T^{\prime}}$ satisfying (1) on $T^{\prime} \subset T$ (Theorem 1 below). This condition is also necessary if the function $f$ satisfies some additional requirements (Theorem 3 ).

2010 Mathematics Subject Classification: Primary 60G07, 60G17; Secondary 60G99.

Key words and phrases: processes with bounded increments, sample continuity.

The paper is in final form and no version of it will be published elsewhere. 
The techniques which are used in this paper stem from the works of Paszkiewicz ([5], [7] or [6]). Therein similar operators, albeit based on conditional $L_{2}$-norms, were invented to give a complete characterization of a.e. convergent orthogonal series (and processes) in $L_{2}$ (cf. our formula (5p). It is also worth noting that much similar operators were also used in 8 for insurance pricing in an unconventional reinsurance model.

The theory of processes with bounded increments on a general compact space $T$, where the right-hand bound in (1) is a metric on $T$, was extensively investigated in e.g. [9], 2, [1. The special case of the unit interval with an additional assumption of continuity of $f$ in (1) was investigated by e.g. [3. This result generalizes the approach used in [4] to investigate a.s. continuity of processes with bounded increments with respect to the metric $\left(d_{\infty}\right)^{\epsilon}, 0<\epsilon<1$, on the unit cube.

2. Criterion of continuity of processes on subsets of unit cube in $\mathbb{R}^{\eta}$. In order to present the crucial characterization of sets $T \subset[0,1]^{\eta}$ we will define the sequence of sets $\Delta_{i}=\Delta_{i}^{T}, i \leq 0$ determined by $T$. We will omit the superscript whenever it does not cause ambiguity.

For any $i \geq 0$ and $0 \leq n<2^{i}-1$ let $P_{n}^{i}=\left[n 2^{-i},(n+1) 2^{-i}\right)$ and $P_{2^{i}-1}^{i}=\left[1-2^{-i}, 1\right]$. We consider families of dyadic ( $i$-atomic) cubes in $[0,1]^{\eta}$, i.e.

$$
\mathcal{F}_{i}^{0}=\left\{P_{n^{1}}^{i} \times \ldots \times P_{n^{\eta}}^{i}: 0 \leq n^{k}<2^{i}, 1 \leq k \leq \eta\right\}, \quad i \geq 0 .
$$

Moreover we will also consider the $\sigma$-fields

$$
\mathcal{F}_{i}=\sigma\left(\mathcal{F}_{i}^{0}\right), \quad i \geq 0
$$

and finally we define for $T \subset[0,1]^{\eta}$

$$
\Delta_{i}=\Delta_{i}^{T}=\bigcap\left\{Z \in \mathcal{F}_{i}: T \subset Z\right\}, \quad i \geq 0 .
$$

For $h \in L^{p}\left([0,1]^{\eta}\right)$ we will use an unusual but convenient notation for the conditional $L_{p}$-norm, i.e.

$$
\|h\|_{p, i}=\left(\mathbb{E}\left(|h|^{p} \mid \mathcal{F}_{i}\right)\right)^{\frac{1}{p}}, i \geq 1 ; \quad\|h\|_{p, 0}:=\|h\|_{p}=\sqrt[p]{\mathbb{E}|h|^{p}} .
$$

The expectations are taken with respect to Lebesgue measure in $[0,1]^{\eta}$.

Our criterion of sample continuity is based on so-called Paszkiewicz-type operators associated with sets $T \subset[0,1]^{\eta}$. Thus to formulate Theorems 1 (below) and 3 , which constitute the main result of the paper, we need to define for any integer $i \geq 0$ the operators

$$
V_{i}^{T} h=2^{i \eta / p} f\left(2^{-i}\right) \mathbb{I}_{\Delta_{i}^{T}}+\|h\|_{p, i}, \quad \text { for } h \in L^{p}\left([0,1]^{\eta}\right) .
$$

Once again we will omit the superscript $T$ whenever it is clear what set determines the operators in question. A basic observation is that those operators are positive and increasing with respect to $T$ and with respect to positive arguments $h$.

Theorem 1. Let $T_{0} \subset[0,1]^{\eta}$ and $V_{i}=V_{i}^{T_{0}}, i \geq 0$ be the operator associated with the set $T_{0}$ by (5). If

$$
\lim _{n \rightarrow \infty} V_{0} \ldots V_{n} 0<\infty
$$

then for every countable $T \subset T_{0}$ and any process $\left(X_{t}\right)_{t \in T}$ with bounded increments on $T$ (cf. (1) $\left(X_{t}\right)_{t \in T}$ is a.s. path continuous. 
Before we present the proof of Theorem 1 let us introduce the following lemma

Lemma 2. Let $T \subset[0,1]^{\eta}$ be countable and the operators $V_{i}=V_{i}^{T}, i \geq 0$ be given by (5). If $\left(X_{t}\right)_{t \in T}$ is a process with bounded increments on $T, k \geq 0$ and $B(t, \varepsilon)$ denotes a $\varepsilon$-ball in $\left(T, d_{\infty}\right)$ then for any $t \in T$

$$
\left\|\sup _{s \in B\left(t, 2^{-k}\right)}\left|X_{t}-X_{s}\right|\right\|_{p} \leq 4^{\eta} \cdot \lim _{n \rightarrow \infty}\left\|V_{k} \ldots V_{n} 0\right\|_{p}+2^{\eta} f\left(2^{-k}\right) .
$$

Proof. Fix a point $t \in T$. First let us notice that since $\left(X_{s}\right)_{s \in T}$ is separable we have

$$
\left\|\sup _{s \in B\left(t, 2^{-k}\right)}\left|X_{t}-X_{s}\right|\right\|_{p}=\sup _{F \subset T: F \text { finite }}\left\|\sup _{s \in B\left(t, 2^{-k}\right) \cap F}\left|X_{t}-X_{s}\right|\right\|_{p} .
$$

Let $F$ be a finite subset of $T$ such that $t \in F$ and let $i_{0}>k$ be an integer large enough so that $\mathcal{F}_{i_{0}}$ separates the points of $F$ e.g. $i_{0}$ satisfying $2^{-i_{0}}<\min _{s, u \in F} d_{\infty}(s, u)$. For any $i \leq i_{0}$, and for any $\delta_{i} \in \mathcal{F}_{i}^{0}$ (cf. (2)) such that $\delta_{i} \cap F \neq \emptyset$ let us fix an element $t_{\delta_{i}} \in \delta_{i} \cap F$.

Obviously $\left\|\max _{s \in \delta_{i_{0}} \cap F}\left|X_{s}-X_{t_{\delta_{i_{0}}}}\right|\right\|_{p}=0$ for all $\delta_{i_{0}} \in \mathcal{F}_{i}^{0}, \delta_{i_{0}} \cap F \neq \emptyset$. Let us assume that for some $i<i_{0}$ and all $\delta_{i+1} \in \mathcal{F}_{i+1}^{0}, \delta_{i+1} \cap F \neq \emptyset$,

$$
\left\|\max _{s \in \delta_{i+1} \cap F} \mid X_{s}-X_{t_{\delta_{i+1}}}\right\|\left\|_{p} \leq 2^{\eta} \cdot\right\| \mathbb{I}_{\delta_{i+1}} V_{i+1} \ldots V_{i_{0}} 0 \|_{p} .
$$

Then, for any $\delta_{i} \in \mathcal{F}_{i}^{0}$ we have the estimate

$$
\begin{aligned}
\left\|\max _{s \in \delta_{i} \cap F}\left|X_{s}-X_{t_{\delta_{i}}}\right|\right\|_{p} & \|\| \max _{\substack{\delta_{i+1} \in \mathcal{F}_{i+1}^{0} \\
\delta_{i+1} \subset \delta_{i}, \delta_{i+1} \cap F \neq \emptyset}}\left|X_{t_{\delta_{i+1}}}-X_{t_{\delta_{i}}}\right|\left\|_{p}+\right\| \max _{\substack{\delta_{i+1} \in \mathcal{F}_{i+1}^{0} \\
\delta_{i+1} \subset \delta_{i}, \delta_{i+1} \cap F \neq \emptyset}} \max _{s \in \delta_{i+1} \cap F}\left|X_{s}-X_{t_{\delta_{i+1}}}\right| \|_{p} \\
& \leq 2^{\eta} f\left(2^{-i}\right)+\left(\sum_{\delta_{i+1} \in \mathcal{F}_{i+1}^{0}, \delta_{i+1} \subset \delta_{i}}\left\|\max _{s \in \delta_{i+1} \cap F}\left|X_{s}-X_{t_{\delta_{i+1}}}\right|\right\|_{p}^{p}\right)^{\frac{1}{p}} \\
& \leq 2^{\eta} f\left(2^{-i}\right)+2^{\eta} \cdot\left(\sum_{\delta_{i+1} \in \mathcal{F}_{i+1}^{0}, \delta_{i+1} \subset \delta_{i}}\left\|\mathbb{I}_{\delta_{i+1}} V_{i+1} \ldots V_{i_{0}}\right\|_{p}^{p}\right)^{\frac{1}{p}} \\
& =2^{\eta}\left(\left\|2^{i \eta} f\left(2^{-i}\right) \mathbb{I}_{\delta_{i}}\right\|_{p}+\left\|\mathbb{I}_{\delta_{i}}\right\| V_{i+1} \ldots V_{i_{0}} 0\left\|_{p, i}\right\|_{p}\right)=2^{\eta}\left\|\mathbb{I}_{\delta_{i}} V_{i} \ldots V_{i_{0}} 0\right\|_{p} .
\end{aligned}
$$

Finally, by induction and a similar estimate we have

$$
\begin{aligned}
& \left\|\sup _{s \in B\left(t, 2^{-k}\right) \cap F}\left|X_{t}-X_{s}\right|\right\|_{p} \\
& \leq\left\|\underset{\substack{\delta_{k+1} \cap B\left(t, 2^{-k}\right) \neq \emptyset \\
\delta_{k+1} \in \mathcal{F}_{k+1}^{0}, \delta_{k+1} \cap F \neq \emptyset}}{ }\left|X_{t_{\delta_{k+1}}}-X_{t}\right|\right\|_{p}+\left\|\max _{\substack{\delta_{k+1} \cap B\left(t, 2^{-k}\right) \neq \emptyset \\
\delta_{k+1} \in \mathcal{F}_{k+1}^{0}}} \max _{s \in \delta_{k+1} \cap F}\left|X_{s}-X_{t_{\delta_{k+1}}}\right|\right\|_{p} \\
& \leq 2^{\eta} f\left(2^{-k}\right)+4^{\eta}\left\|\mathbb{I}_{B\left(t, 2^{-k-1}\right)} V_{k+1} \ldots V_{i_{0}} 0\right\|_{p} \\
& \leq 4^{\eta} \cdot \lim _{n \rightarrow \infty}\left\|\mathbb{I}_{B\left(t, 2^{-k-1}\right)} V_{k} \ldots V_{n} 0\right\|_{p}+2^{\eta} f\left(2^{-k}\right) .
\end{aligned}
$$

Proof of Theorem 1. Let $t$ be a point in $T$. Let $k>0$ be an integer. Notice that $f\left(2^{-k}\right)=$ $\left\|V_{k}^{\{t\}} 0\right\|_{p}$. By (6) we have for $\delta_{k} \in \mathcal{F}_{k}^{0}$

$$
\lim _{n \rightarrow \infty}\left\|\mathbb{I}_{\delta_{k}} V_{k} \ldots V_{n} 0\right\|_{p} \rightarrow 0 \text { for } k \rightarrow \infty
$$


thus by (6) we can choose an increasing sequence of integers $\left(k_{i}\right)_{i \in \mathbb{N}}$ such that

$$
\sum_{i \in \mathbb{N}}\left(f\left(2^{-k_{i}}\right)+\lim _{n \rightarrow \infty}\left\|\mathbb{I}_{B\left(t, 2^{-k_{i}-1}\right)} V_{k_{i}} \ldots V_{n} 0\right\|_{p}\right)<\infty
$$

With $B(t, \varepsilon)$ denoting the $d_{\infty}$-ball with centre at $t$ and radius $\varepsilon$, since obviously $0 \leq V_{i}^{T} \leq V_{i}=V_{i}^{T_{0}}, i \geq 0$, by Lemma 2 we have

$$
\left\|\sup _{s \in B\left(t, 2^{-k_{i}}\right)}\left|X_{s}-X_{t}\right|\right\|_{p} \leq 2^{\eta} f\left(2^{-k_{i}}\right)+4^{\eta} \lim _{n \rightarrow \infty}\left\|\mathbb{I}_{B\left(t, 2^{-k_{i}-1}\right)} V_{k_{i}} \ldots V_{n} 0\right\|_{p} .
$$

This implies that $\sum_{i \in \mathbb{N}} \mathbb{E} \sup _{s \in B\left(t, 2^{-k_{i}}\right)}\left|X_{s}-X_{t}\right|<\infty$, which (by properties of monotonic sequences) yields

$$
\sup _{s \in B\left(t, 2^{-k_{i}}\right)}\left|X_{s}-X_{t}\right| \rightarrow 0 \quad \text { a.s. with } i \rightarrow \infty .
$$

Thus $\left(X_{s}\right)_{s \in T}$ is a.s. continuous in $t \in T$.

TheOREM 3. Let $T_{0} \subset[0,1]^{\eta}$ be a closed set and $V_{i}, i \geq 0$ be the operators associated with the set $T_{0}$ by (5). If the non-decreasing function $f$ introduced in (1) satisfies an additional growth condition, namely for some constant $C>0$

$$
\sum_{k=n}^{\infty} f\left(2^{-k}\right)+\sum_{k=0}^{n-1} 2^{k-n} f\left(2^{-k}\right) \leq C \cdot f\left(2^{-n}\right), \quad n \geq 0,
$$

then whenever

$$
\lim _{n \rightarrow \infty} V_{0} \ldots V_{n} 0=\infty
$$

there exists a countable $T \subset T_{0}$ and a process $\left(X_{t}\right)_{t \in T}$ with bounded increments on $T$ which is a.s. discontinuous at some $t_{0} \in T$.

It is convenient to first prove the following lemma.

Lemma 4. Let $T \subset[0,1]^{\eta}$ and let $V_{i}=V_{i}^{T}, i \geq 0$ be the operator associated with the set $T$ by (5). Assume that a non-decreasing $f$ satisfies (7), for some $C>0$. For any integers $j_{0}<i_{0}$ and $\delta_{j_{0}} \in \mathcal{F}_{j_{0}}^{0}$ there exists a finite $F \subset \delta_{j_{0}} \cap T$ and a process $\left(X_{t}\right)_{t \in F}$ with bounded increments (cf. (1)) for which

$$
\left\|\max _{t \in F}\left|X_{t}\right|\right\|_{p} \geq \frac{1}{K_{C, \eta}}\left\|\mathbb{I}_{\delta_{j_{0}}} V_{j_{0}} \ldots V_{i_{0}} 0\right\|_{p}, \quad \max _{t \in F}\left\|X_{t}\right\|_{p} \leq K_{C, \eta} f\left(2^{-j_{0}}\right),
$$

where $K_{C, \eta}>0$ is a constant.

Proof. Fix integers $j_{0}<i_{0}$ and a set $\delta_{j_{0}} \in \mathcal{F}_{j_{0}}^{0}$. Let $F \subset T \cap \delta_{j_{0}}$ be a finite set satisfying $\Delta_{i_{0}}^{T} \cap \delta_{j_{0}}=\Delta_{i_{0}}^{F}$ (it is enough to choose one point from each nonempty set in the family $\left.\left(T \cap \delta_{j_{0}} \cap \delta\right)_{\delta \in \mathcal{F}_{i_{0}}^{0}}\right)$, according to 4 .

Now by induction we define sequences of variables $\xi_{k}$ and $X_{t}^{k}, t \in F, j_{0} \leq k \leq i_{0}$ on the probability space $[0,1]^{\eta}$. For $t \in F$ let

$$
X_{t}^{i_{0}}(\omega)=\sum_{\delta \in \mathcal{F}_{i_{0}}^{0}} 2^{i_{0} \eta / p} f\left(2^{-i_{0}}\right)\left(1-2^{i_{0}} d(t, \delta)\right)^{+} \mathbb{I}_{\delta}(\omega) \mathbb{I}_{\Delta_{i_{0}}^{F}}(\omega),
$$


where $A^{+}:=\max \{A, 0\}, A \in \mathbb{R}$. Moreover let $\xi_{i_{0}}=\left\|\mathbb{I}_{\Delta_{i_{0}}}\right\|_{p, i_{0}-1}^{-1} \mathbb{I}_{\Delta_{i_{0}}}$ with $0 / 0:=0$. Then inductively we define for $j_{0} \leq k<i_{0}$

$$
\begin{aligned}
& X_{t}^{k}(\omega)=X_{t}^{k+1}(\omega)+\sum_{\delta \in \mathcal{F}_{k}^{0}} 2^{k \eta / p} f\left(2^{-k}\right)\left(1-2^{k} d(t, \delta)\right)^{+} \mathbb{I}_{\delta}(\omega) \xi_{k+1}(\omega), \\
& \xi_{k}(\omega)=\frac{\max _{t \in F \cap \delta_{k-1}} X_{t}^{k}(\omega)}{\left\|\max _{t \in F \cap \delta_{k-1}} X_{t}^{k}\right\|_{p, k-1}}, \quad \text { for } \delta_{k-1} \in \mathcal{F}_{k-1}^{0}, \quad \omega \in \delta_{k-1},
\end{aligned}
$$

with $0 / 0:=0$ and $\xi_{k}=0$ if $F \cap \delta_{k-1}=\emptyset$.

For the process $X_{t}^{j_{0}}, t \in F$ we have $\max _{t \in F}\left\|X_{t}^{j_{0}}\right\|_{p} \leq 3^{\eta} C f\left(2^{-j_{0}}\right)$ since by an easy computation using (7) for any $j_{0} \leq k \leq i_{0}$ and $t \in F$ we have

$$
\left\|X_{t}^{k}\right\|_{p} \leq \sum_{i=k}^{i_{0}} \sum_{\delta \in \mathcal{F}_{k}^{0}}\left\|2^{i \eta / p} f\left(2^{-i}\right)\left(1-2^{i} d(t, \delta)\right)^{+} \mathbb{I}_{\delta}\right\|_{p} \leq 3^{\eta} \sum_{i=k}^{i_{0}} f\left(2^{-i}\right) \leq 3^{\eta} C \cdot f\left(2^{-k}\right)
$$

(notice that for any $i \geq 0$ the term $\left(1-2^{i} d(t, \delta)\right)^{+}$is positive for at most $3^{\eta}$ sets $\delta$ in $\left.\mathcal{F}_{i}^{0}\right)$

To demonstrate that the first stipulation in 8 is also satisfied (up to some constant factor) for the process $\left(X_{t}^{j_{0}}\right)_{t \in F}$ we will inductively show that for any $\delta_{j_{0}} \in \mathcal{F}_{j_{0}}^{0}$ we have

$$
\left\|\max _{t \in F} X_{t}^{j_{0}}\right\|_{p} \geq\left\|\mathbb{I}_{\delta_{j_{0}}} \max _{t \in F \cap \delta_{j_{0}}} X_{t}^{j_{0}}\right\|_{p} \geq\left\|\mathbb{I}_{\delta_{j_{0}}} V_{j_{0}} \ldots V_{i_{0}} 0\right\|_{p}
$$

Assume that for some $j_{0} \leq k \leq i_{0}$ and any $\delta_{k} \in \mathcal{F}_{k}^{0}$ we have

$$
\left\|\mathbb{I}_{\delta_{k}} \max _{t \in F \cap \delta_{k}} X_{t}^{k}\right\|_{p} \geq\left\|\mathbb{I}_{\delta_{k}} V_{k} \ldots V_{i_{0}} 0\right\|_{p} .
$$

Notice that this is indeed true for $k=i_{0}$, namely

$$
\left\|\mathbb{I}_{\delta_{i_{0}}} \max _{t \in F \cap \delta_{i_{0}}} X_{t}^{i_{0}}\right\|_{p}=2^{i_{o} \eta / p} f\left(2^{-i_{0}}\right) \cdot\left\|\mathbb{I}_{\delta_{i_{0}} \cap \Delta_{i_{0}}^{F}}\right\|_{p}=\left\|\mathbb{I}_{\delta_{i_{0}}} V_{i_{0}} 0\right\|_{p}
$$

for any $\delta_{i_{0}} \in \mathcal{F}_{i_{0}}^{0}$. For any $\delta_{k-1} \subset \Delta_{k-1}^{F}=\Delta_{k-1}^{T}, \delta_{k-1} \in \mathcal{F}_{k-1}^{0}$, by collinearity of $\max _{t \in F \cap \delta_{k-1}} X_{t}^{k}$ and $\xi_{k}$ on $\delta_{k-1}$, the following estimate holds

$$
\begin{gathered}
\left\|\mathbb{I}_{\delta_{k-1}} \max _{t \in F \cap \delta_{k-1}} X_{t}^{k-1}\right\|_{p}=\left\|\mathbb{I}_{\delta_{k-1}}\left[\max _{t \in F \cap \delta_{k-1}}\left(X_{t}^{k}+2^{(k-1) \eta / p} f\left(2^{-(k-1)}\right) \xi_{k} \mathbb{I}_{\delta_{k-1}}\right)\right]\right\|_{p} \\
\geq\left\|\left(\left\|\sum_{\delta_{k} \subset \delta_{k-1}, \delta_{k} \in \mathcal{F}_{k}^{0}} \mathbb{I}_{\delta_{k}} \max _{t \in F \cap \delta_{k}} X_{t}^{k}\right\|_{p, k-1}+\left\|2^{(k-1) \eta / p} f\left(2^{-(k-1)}\right) \xi_{k} \mathbb{I}_{\delta_{k-1}}\right\| \|_{p, k-1}\right) \mathbb{I}_{\delta_{k-1}}\right\|_{p} \\
=\left\|\left(\sqrt[p]{\sum_{\delta_{k} \subset \delta_{k-1}, \delta_{k} \in \mathcal{F}_{k}^{0}} 2^{(k-1) \eta}\left\|\mathbb{I}_{\delta_{k}} \max _{t \in F \cap \delta_{k}} X_{t}^{k}\right\|_{p}^{p}}+2^{(k-1) \eta / p} f\left(2^{-(k-1)}\right)\right) \mathbb{I}_{\delta_{k-1}}\right\|_{p} \\
\geq\left\|\left(\sqrt[p]{\sum_{\delta_{k} \subset \delta_{k-1}, \delta_{k} \in \mathcal{F}_{k}^{0}} 2^{(k-1) \eta}\left\|\mathbb{I}_{\delta_{k}} V_{k} \ldots V_{i_{0}} 0\right\|_{p}^{p}}+2^{(k-1) \eta / p} f\left(2^{-(k-1)}\right)\right) \mathbb{I}_{\delta_{k-1}}\right\|_{p} \\
=\left\|\mathbb{I}_{\delta_{k-1}} V_{k-1} V_{k} \ldots V_{i_{0}} 0\right\|_{p} .
\end{gathered}
$$

Now, let us assume that $s, t \in F$ and let $j$ be an integer satisfying $2^{j} \leq d(s, t) \leq 2^{j+1}$. By (7) we have

$$
f\left(2^{-j}\right) \leq f(d(s, t)) \leq 2 C \cdot f\left(2^{-j}\right) .
$$

We will show that $\left\|X_{t}-X_{s}\right\|_{p}$ is also of order $f\left(2^{-j}\right)$. 
We have

$$
\left\|X_{t}^{j_{0}}-X_{s}^{j_{0}}\right\|_{p} \leq\left\|X_{t}^{j_{0}}-X_{t}^{j}+X_{s}^{j}-X_{s}^{j_{0}}\right\|_{p}+\left\|X_{t}^{j}\right\|_{p}+\left\|X_{s}^{j}\right\|_{p}
$$

and

$$
\begin{aligned}
& \left\|X_{t}^{j_{0}}-X_{t}^{j}+X_{s}^{j}-X_{s}^{j_{0}}\right\|_{p} \\
& \leq\left\|\sum_{k=j_{0}}^{j-1} \sum_{\delta \in \mathcal{F}_{k}^{0}}\left|\left(1-2^{k} d(t, \delta)\right)^{+}-\left(1-2^{k} d(s, \delta)\right)^{+}\right| 2^{k \eta / p} f\left(2^{-k}\right) \xi_{k} \mathbb{I}_{\delta}\right\|_{p} \\
& \leq \sum_{k=j_{0}}^{j-1} 2 \cdot 3^{\eta} d(t, s) \cdot 2^{-k} f\left(2^{-k}\right)
\end{aligned}
$$

since the expression $\left|\left(1-2^{k} d(t, \delta)\right)^{+}-\left(1-2^{k} d(s, \delta)\right)^{+}\right|$is positive for at most $2 \cdot 3^{\eta}$ sets $\delta \in \mathcal{F}_{k}^{0}, k \geq 0$, and it does not exceed $2^{k}|d(t, \delta)-d(s, \delta)| \leq 2^{k} d(t, s)$. By (7), 10, we further obtain

$$
\sum_{k=j_{0}}^{j-1} 2 \cdot 3^{\eta} d(t, s) \cdot 2^{-k} f\left(2^{-k}\right) \leq 2 \cdot 3^{\eta} \sum_{k=j_{0}}^{j-1} 2^{-l} 2^{k} f\left(2^{-k}\right) \leq 2 \cdot 3^{\eta} C \cdot f\left(2^{-j}\right) .
$$

This, together with (9), implies that $\left(\left(4 C 3^{\eta}\right)^{-1} X_{t}^{j_{0}}\right)_{t \in F}$ has bounded increments. Thus it is enough to take $X_{t}=\left(4 C 3^{\eta}\right)^{-1} X_{t}^{j_{0}}$, for $t \in F$, and $K_{\eta, C}=4 C 3^{\eta}$.

Proof of Theorem 3 Recall that $V_{k}=V_{k}^{T_{0}}, k \geq 0$, are operators associated with the set $T_{0}$. Since $\lim _{n \rightarrow \infty}\left\|V_{0}^{T_{0}} \ldots V_{n}^{T_{0}} 0\right\|_{p}=\infty$ and

$$
\left\|V_{k} \ldots V_{n} 0\right\|_{p, k} \leq\left\|V_{k} \ldots V_{k^{\prime}} 0\right\|_{p, k}+\left\|V_{k^{\prime}+1} \ldots V_{n} 0\right\|_{p, k}, \quad 0 \leq k \leq k^{\prime}<n,
$$

by subadditivity of conditional norms, we can choose a sequence $\left(\tilde{\delta}^{k}\right)_{k \geq 0}$ of sets such that $\tilde{\delta}^{k} \in \mathcal{F}_{k}^{0}($ cf. $(2)) ; \tilde{\delta}^{k} \subset \tilde{\delta}^{k+1}, k \geq 0$ and

$$
\lim _{n \rightarrow \infty}\left\|I_{\tilde{\delta}^{k}} V_{k}^{T_{0}} \ldots V_{n}^{T_{0}} 0\right\|_{p}=\infty .
$$

Let us consider the point $t_{0} \in T_{0}=\operatorname{cl}\left(T_{0}\right)$, where $\operatorname{cl}(\cdot)$ denotes closure of sets, satisfying $t_{0} \in \bigcap_{k>0} \operatorname{cl}\left(\tilde{\delta}^{k+1}\right)$. Let $m_{0}$ be an integer such that all binary-rational coordinates of $t_{0}$ are multiples of $2^{-m_{0}}$. If all coordinates of $t_{0}$ are binary-irrational put $m_{0}=0$. It is easily seen that for all $k>m_{0}$ there exist $k^{\prime}>k$ such that $d_{\infty}\left(\tilde{\delta}^{k^{\prime \prime}}, \tilde{\delta}^{m_{0}} \backslash \tilde{\delta}^{k}\right)>0$, for all $k^{\prime \prime} \geq k^{\prime}$. Namely, let $k>m_{0}$. If for every $k^{\prime}>k$ we have $d\left(\tilde{\delta}^{k^{\prime}}, \tilde{\delta}^{m_{0}} \backslash \tilde{\delta}^{k}\right)=0$ then $d\left(t_{0}, \tilde{\delta}^{m_{0}} \backslash \tilde{\delta}^{k}\right) \leq$ $\lim _{k^{\prime} \rightarrow \infty} \operatorname{diam}\left(\tilde{\delta}^{k^{\prime}}\right)=0$. Thus, since $t_{0} \in \tilde{\delta}^{k}$ and $t_{0} \in \operatorname{cl}\left(\tilde{\delta}^{m_{0}} \backslash \tilde{\delta}^{k}\right)$ a coordinate of $t_{0}$ which is not a multiple of $2^{-m_{0}}$ is a multiple of $2^{-k}$. This is a contradiction. Obviously $d\left(\tilde{\delta}^{m_{0}} \backslash \tilde{\delta}^{k}\right) \leq d\left(\tilde{\delta}^{k^{\prime \prime}}, \tilde{\delta}^{m_{0}} \backslash \tilde{\delta}^{k}\right)$, for $k^{\prime \prime}>k^{\prime}$.

Let us set $k_{0}=m_{0}$. Assume that $k_{i}, m_{i}$ for some $i \geq 0$ are defined. Then, since for $m \geq k_{i}$ by e.g. the monotone convergence theorem

$$
\lim _{m \rightarrow \infty}\left\|\mathbb{I}_{\tilde{\delta}^{k_{i}}} V_{k_{i}}^{T_{0} \backslash \tilde{\delta}^{m}} \ldots V_{n}^{T_{0} \backslash \tilde{\delta}^{m}} 0\right\|_{p}=\left\|\mathbb{I}_{\tilde{\delta}^{k_{i}}} V_{k_{i}}^{T_{0}} \ldots V_{n}^{T_{0}} 0\right\|_{p},
$$

the condition 11 implies that we can choose integers $k_{i+1}>n_{i}$ so that

$$
\left\|\mathbb{I}_{\tilde{\delta}^{k_{i}}} V_{k_{i}}^{T_{0} \backslash \tilde{\delta}^{k_{i+1}}} \ldots V_{n_{i}}^{T_{0} \backslash \tilde{\delta}^{k_{i+1}}} 0\right\|_{p}>2 \cdot K_{\eta, C}+K_{\eta, C}^{2} f\left(2^{-k_{i}}\right),
$$

as well as

$$
d_{\infty}\left(\tilde{\delta}^{k_{i+1}}, \tilde{\delta}^{k_{0}} \backslash \delta^{n_{i}}\right)>0 .
$$


By Lemma 4 for each $i \geq 0$ there exists a finite subset of $F_{i}$ of $T_{0} \cap \tilde{\delta}^{k_{i}} \backslash \tilde{\delta}^{n_{i}}$ and a process $\left(\tilde{X}_{t}^{i}\right)_{t \in F_{i}}$ with bounded increments such that

$$
\left\|\max _{t \in F_{i}} \tilde{X}_{t}^{i}\right\|_{p} \geq K_{\eta, C} f\left(2^{-k_{i}}\right)+2 \max _{t \in F_{i}}\left\|\tilde{X}_{t}^{i}\right\|_{p} \leq K_{\eta, C} f\left(2^{-k_{i}}\right) .
$$

Let us fix $\tau(i)$ in $F_{i}$ such that $d\left(t_{0}, \tau(i)\right)=d\left(t_{0}, F_{i}\right)$. By taking $\bar{X}_{t}^{i}=\tilde{X}_{t}^{i}-\tilde{X}_{\tau(i)}^{i}$ or $\bar{X}_{t}^{i}=\tilde{X}_{\tau(i)}^{i}-\tilde{X}_{t}^{i}$ we obtain a process $\bar{X}_{t}^{i}$ with bounded increments for which

$$
\left\|\max _{t \in F_{i}}\left|\bar{X}_{t}^{i}\right|\right\|_{p} \geq 1, \quad \bar{X}_{\tau(i)}^{i}=0 \text {. }
$$

Set $\zeta_{i}=\max _{t \in F_{i}}\left|\bar{X}_{t}^{i}\right|$. It is a standard argument that by taking $X_{t}^{i}=\bar{X}_{t}^{i} \zeta_{i}^{-1 / p}, t \in F_{i}$ on the probability space $\left(\left\{\zeta_{i}>0\right\}, \Lambda_{i}\right)$, where $\mathrm{d} \Lambda_{i}=\left\|\zeta_{i}\right\|_{p}^{-p} \zeta_{i} \mathrm{~d} \lambda$ we obtain a process $\left(X_{t}^{i}\right)_{t \in F_{i}}$ with bounded increments and

$$
X_{\tau(i)}^{i}=0 \text { and } \max _{t \in F_{i}}\left|X_{t}^{i}\right| \geq 1 \quad \text { a.e. }
$$

Let $T=\bigcup_{i=0}^{\infty} F_{i} \cup\left\{t_{0}\right\}$ and $\left(X_{t}\right)_{t \in T}$ be a process given by the following:

- $\left(X_{t}\right)_{t \in F_{i}}$ and $\left(X_{t}^{i}\right)_{t \in F_{i}}$ have the same distribution, for $i \geq 0$,

$-X_{t_{0}}=0$ a.e.

Let $\left(t_{n}\right)_{n \in \mathbb{N}}$ be a sequence of all elements of $\bigcup_{k=0}^{\infty} F_{i}$. Naturally $t_{n} \rightarrow t_{0}$ with $n \rightarrow \infty$. Moreover $\min _{i \geq 0} \max _{t \in F_{i}}\left|X_{t}\right| \geq 1$ on some set of full measure. Thus almost surely the sequence $\left(\left|X_{t_{n}}-X_{t_{0}}\right|\right)_{n \in N}$ attains a value greater than or equal to 1 for an infinite number of indices.

It suffices to show that $\left(\frac{1}{8 C^{2}+1} X_{t}\right)_{t \in T}$ has bounded increments. Let $t, s \in T$. If $t \in F_{i}$, $s \in F_{j} \subset \tilde{\delta}^{k_{j}}$ for some $j>i \geq 0$ then by 13 .

$$
2^{-k_{j}} \leq d_{\infty}\left(\tilde{\delta}^{k_{j}}, \tilde{\delta}^{k_{0}} \backslash \tilde{\delta}^{k_{i}}\right) \leq d_{\infty}\left(F_{j}, F_{i}\right) \leq d_{\infty}(t, s),
$$

since for arbitrary $k \geq 0, A, B \in \mathcal{F}_{k}$ the quantity $d_{\infty}(A, B)$ is a multiple of $2^{-k}$. We also have

$$
\begin{aligned}
d_{\infty}(t, \tau(i)) & \leq d(t, s)+d\left(s, t_{0}\right)+d\left(t_{0}, \tau(i)\right) \leq d(t, s)+2^{-k_{j}}+d\left(t_{0}, t\right) \\
& \leq d(t, s)+2^{-k_{j}}+d(t, s)+2^{-k_{j}} \leq 4 d(t, s)
\end{aligned}
$$

and by 7 )

$$
\begin{aligned}
\left\|X_{t}-X_{s}\right\|_{p} & \leq\left\|X_{t}-X_{\tau(i)}\right\|_{p}+\left\|X_{s}\right\|_{p} \leq f(4 d(t, s))+f\left(2^{-k_{j}}\right) \\
& \leq f\left(4 \cdot 2^{\left\lceil\log _{2} d(t, s)\right\rceil}\right)+f(d(t, s)) \leq 4 C f\left(2^{\left\lceil\log _{2} d(t, s)\right\rceil}\right)+f(d(t, s)) \\
& \leq 8 C^{2} f(d(t, s))+f(d(t, s)) \leq\left(8 C^{2}+1\right) f\left(d_{\infty}(t, s)\right) .
\end{aligned}
$$

\section{References}

[1] W. Bednorz, A theorem on majorizing measures, Ann. Probab. 34, no. 5 (2006), 1771-1781.

[2] W. Bednorz, Majorizing measures on metric spaces, preprint.

[3] N. Kôno, Best possibility of an integral test for sample continuity of $L_{p}$-processes $(p>2)$, Proc. Japan. Acad. Ser. A 54 (1978). 
[4] J. Olejnik, On a construction of a majorizing measure on subsets of $R^{n}$ with special metrics, Studia Mathematica 197, no. 1 (2010).

[5] A. Paszkiewicz, On complete characterization of coefficients of a.e. convergent orthogonal series, Preprint, University of Łódź, Faculty of Mathematics and Computer Science (2008/03).

[6] A. Paszkiewicz, The explicit characterization of coefficients of a.e. orthogonal series, Comptes Rendus Mathématique 347, issues 19-20 (2009), 1213-1216.

[7] A. Paszkiewicz, A complete characterization of coefficients of a.e. orthogonal series and majorizing measures, Inventiones Mathematicae 180, no. 1 (2010).

[8] A. Paszkiewicz and J. Olejnik, Reinsurance-a new approach, this volume, 139-151.

[9] M. Talagrand, Sample boundedness of stochastic processes under increment conditions, Ann. Probab. 18 (1990), 1-49. 